\title{
SMOOTH CONVEX $t$-NORMS DO NOT EXIST
}

\author{
C. ALSINA AND M. S. TOMAS
}

(Communicated by Richard R. Goldberg)

ABStraCt. We show that smooth convex $t$-norms do not exist.

Let $T$ be a $t$-norm, i.e., a binary operation on $[0,1]$ which is associative, commutative, nondecreasing in each place, and has 1 as a unit element. A $t$-norm $T$ is convex if, for all $\lambda$ in $[0,1]$ and for all $a, b, c, d$ in $[0,1], T$ satisfies:

$$
T(\lambda a+(1-\lambda) b, \lambda c+(1-\lambda) d) \leq \lambda T(a, c)+(1-\lambda) T(b, d) .
$$

Convex $t$-norms play a fundamental role in some studies of products of probabilistic metric spaces (see $[2,6]$ ). An example of continuous convex $t$-norm is $W(x, y)=$ $\operatorname{Max}(x+y-1,0)$ and a discontinuous example is $Z(x, y)=0$ whenever $(x, y)$ is in $[0,1) \times[0,1)$ and $Z(x, y)=W(x, y)$ otherwise.

All other examples of continuous convex $t$-norms are closely related to $W$. Consequently, it is natural to ask whether smooth convex $t$-norms can exist. Our aim in this paper is to show that they cannot. To this end we begin with the following

LEMMA 1. If $T$ is a continuous convex $t$-norm, then: (i) $T \leq W$; (ii) $T$ admits a representation of the form $T(x, y)=f^{(-1)}(f(x)+f(y))$, where $f$ (the additive generator of $T)$ is a continuous strictly decreasing function from $[0,1]$ onto $[0,1]$ such that $f(0)=1, f(1)=0$, and $f^{(-1)}$ is the pseudo-inverse of $f$, i.e., $f^{(-1)}=f^{-1}$ on $[0,1]$ and $f^{(-1)}(x)=0$, whenever $x \geq 1$; (iii) $f$ is concave on $\left[f^{-1}\left(\frac{1}{2}\right), 1\right]$.

ProOF. Since $T$ is a convex $t$-norm, for any $a, b$ and $\lambda$ in $[0,1]$ we have

$$
T(\lambda a+(1-\lambda) b, \lambda b+(1-\lambda) a) \leq \lambda T(a, b)+(1-\lambda) T(b, a)=T(a, b) .
$$

Suppose $x, y$ in $[0,1]$ are such that $x+y>1$. Then substituting $a=x+y-1$, $b=1$, and $\lambda=(1-x) /(2-x-y)$ in (1) yields $T(x, y) \leq T(x+y-1,1)=x+y-1$, from which (i) follows. The representation of $T$ follows from the theorem of Ling [5]. Finally, the substitution $\lambda=\frac{1}{2}$ into (1) yields $T\left(\frac{1}{2}(a+b), \frac{1}{2}(a+b)\right) \leq T(a, b)$. Using the representation, for any $a, b$ in $\left[f^{-1}\left(\frac{1}{2}\right), 1\right]$ we have $(a+b) / 2 \geq f^{-1}\left(\frac{1}{2}\right)$ and $2 f((a+b) / 2)<1$. Therefore $f^{-1}(2 f((a+b) / 2))>0$ and we obtain

$$
0<f^{-1}\left(2 f\left(\frac{a+b}{2}\right)\right)=T\left(\frac{a+b}{2}, \frac{a+b}{2}\right) \leq T(a, b)=f^{-1}(f(a)+f(b)),
$$

whence $f((a+b) / 2) \geq \frac{1}{2}(f(a)+f(b))$, i.e., $f$ is concave on $\left[f^{-1}\left(\frac{1}{2}\right), 1\right]$.

DEFINITION 1. A $t$-norm $T$ representable in the form

$$
T(x, y)=f^{(-1)}(f(x)+f(y))
$$

Received by the editors April 2, 1986 and, in revised form, September 15, 1986.

1980 Mathematics Subject Classification (1985 Revision). Primary 39C05, 26B25. 
will be called smooth if $f^{\prime}$ and $f^{\prime \prime}$ exist on $(0,1), f^{\prime}(x) \neq 0$ for all $x$ in $(0,1)$, $\lim _{x \rightarrow 0^{+}} x \cdot f^{\prime}(x)=-\infty$, and $f^{\prime \prime}(x)$ is bounded on an arbitrary left-neighborhood of 1 of the form $(1-\varepsilon, 1)$.

Thus for smooth $t$-norms $T$ the first and second partial derivatives of $T$ exist on $(0,1) \times(0,1)$.

LEMMA 2. If $T(x, y)=f^{(-1)}(f(x)+f(y))$ is a smooth convex $t$-norm, then

$$
\lim _{k \rightarrow 1^{-}} \frac{f^{\prime \prime}(k)}{f^{\prime}(k)^{2}}=0
$$

PROOF. Since $f^{\prime \prime}$ is assumed to be bounded on a neighborhood of 1 , we will prove that $\lim _{k \rightarrow 1^{-}} f^{\prime}(k)=-\infty$. Choose a sequence $\left(y_{n}\right)_{n \in N}$ converging to 0 so that $\left(1-f\left(y_{n}\right)\right) / y_{n}>n$. (This is possible because $\lim _{x \rightarrow 0^{+}} f^{\prime}(x)=-\infty$.) Let $x_{n}=f^{-1}\left(1-f\left(y_{n}\right)\right)$. Since $f\left(x_{n}\right)+f\left(y_{n}\right)=1$ and $T \leq W$ we have

$$
0=T\left(y_{n}, 1-y_{n}\right)=f^{(-1)}\left(1-f\left(x_{n}\right)+f\left(1-y_{n}\right)\right)
$$

i.e., $1-f\left(x_{n}\right)+f\left(1-y_{n}\right) \geq 1$. Hence $1-x_{n} \leq y_{n}$ and we have

$$
\frac{f\left(x_{n}\right)}{1-x_{n}} \geq \frac{f\left(x_{n}\right)}{y_{n}}=\frac{1-f\left(y_{n}\right)}{y_{n}}>n \text {. }
$$

It follows that

$$
\lim _{x \rightarrow 1^{-}} f^{\prime}(x)=\lim _{n \rightarrow \infty} \frac{f\left(x_{n}\right)}{x_{n}-1}=-\infty .
$$

LEMMA 3. If $g$ is a function from $(0,1)$ into $\mathbf{R}$ satisfying

(i) $g(u) \leq 0$ whenever $u$ is in $\left(0, \frac{1}{2}\right]$;

(ii) $g(u) \leq g(u+v)$ for all $u, v$ in $(0,1)$ with $u+v \leq 1$;

(iii) $g(u) \cdot g(v) \geq(g(u)+g(v)) \cdot g(u+v)$ for all $u, v$ in $(0,1)$ with $u+v \leq 1$;

(iv) $\lim _{h \rightarrow 0^{+}} g(h)$ exists

then $g(x)=0$ for all $x$ in $(0,1)$.

ProOF. Given any $x$ in $\left(0, \frac{1}{2}\right]$ the substitution $u=v=x$ into (iii) yields $g(x)^{2} \geq 2 g(x) g(2 x)$. If $g(x)<0$ then $g(x) \leq 2 g(2 x)$ and by induction

$$
g(2 x) \geq \frac{1}{2} g(x) \geq \frac{1}{2^{(n+1)}} \cdot g\left(\frac{x}{2^{n}}\right)
$$

whence by (iv)

$$
g(2 x) \geq \frac{1}{2} g(x) \geq \lim _{n \rightarrow \infty} \frac{1}{2^{n+1}} \cdot g\left(\frac{x}{2^{n}}\right)=0,
$$

i.e., $g(x) \geq 0$ in contradiction with $g(x)<0$. Therefore by (i) we have $g(x)=0$ whenever $x$ is in $\left(0, \frac{1}{2}\right]$. Now for any $x$ in $(0,1)$ we have by (ii), $g(x / 2)=0 \leq g(x)$. Consequently for any $u, v$ in $(0,1)$ with $u+v \leq 1$ we will have by (iii) and (ii):

$$
g(u) \cdot g(v) \geq(g(u)+g(v)) \cdot g(u+v) \geq(g(u)+g(v)) g(u) .
$$

Thus $0 \geq g(u)^{2}$ and we can conclude $g(u)=0$ for all $u$ in $(0,1)$. We remark that condition (iv) is essential because there are nonzero functions such as $g(x)=-1 / x$ that satisfy (i), (ii), and (iii). 
THEOREM 1. Smooth convex t-norms cannot exist.

ProOF. Let $T$ be a continuous convex $t$-norm. By Lemma 1, $T$ can be represented in the form

$$
T(x, y)=f^{(-1)}(f(x)+f(y))
$$

where $f$ is concave on $\left[f^{-1}\left(\frac{1}{2}\right), 1\right]$. If $T$ is smooth and convex then it is well known [4] that the following conditions hold:

$$
\frac{\partial^{2} T}{\partial x^{2}}(u, v) \geq 0
$$

and

$$
\frac{\partial^{2} T}{\partial x^{2}}(u, v) \cdot \frac{\partial^{2} T}{\partial y^{2}}(u, v)-\left[\frac{\partial^{2} T}{\partial x \partial y}(u, v)\right]^{2} \geq 0
$$

for all $u, v$ in $(0,1)$. Using (2), after some computations we have that for any $x, y$ in $(0,1)$ with $f(x)+f(y)<1$ the inequalities (3) and (4) become

$$
\frac{f^{\prime \prime}(x)}{f^{\prime}(x)^{2}} \leq \frac{f^{\prime \prime}(T(x, y))}{f^{\prime}(T(x, y))^{2}}
$$

and

$$
\frac{f^{\prime \prime}(x) f^{\prime \prime}(y)}{f^{\prime}(x)^{2} f^{\prime}(y)^{2}} \geq \frac{f^{\prime \prime}(x) f^{\prime \prime}(T(x, y))}{f^{\prime}(x)^{2} f^{\prime}(T(x, y))^{2}}+\frac{f^{\prime \prime}(y) f^{\prime \prime}(T(x, y))}{f^{\prime}(y)^{2} f^{\prime}(T(x, y))^{2}} .
$$

Let $f(x)=u$ and $f(y)=v$. Then for $u, v$ in $(0,1)$ with $u+v<1$, we have

$$
\frac{f^{\prime \prime}\left(f^{-1}(u)\right)}{f^{\prime}\left(f^{-1}(u)\right)^{2}} \leq \frac{f^{\prime \prime}\left(f^{-1}(u+v)\right)}{f^{\prime}\left(f^{-1}(u+v)\right)^{2}}
$$

and

$$
\begin{aligned}
& \frac{f^{\prime \prime}\left(f^{-1}(u)\right)}{f^{\prime}\left(f^{-1}(u)\right)^{2}} \cdot \frac{f^{\prime \prime}\left(f^{-1}(v)\right)}{f^{\prime}\left(f^{-1}(v)\right)^{2}} \\
& \quad \geq\left[\frac{f^{\prime \prime}\left(f^{-1}(u)\right)}{f^{\prime}\left(f^{-1}(u)\right)^{2}}+\frac{f^{\prime \prime}\left(f^{-1}(v)\right)}{f^{\prime}\left(f^{-1}(v)\right)^{2}}\right] \cdot \frac{f^{\prime \prime}\left(f^{-1}(u+v)\right)}{f^{\prime}\left(f^{-1}(u+v)\right)^{2}} .
\end{aligned}
$$

Define $g$ from $(0,1)$ into $\mathbf{R}$ by $g(z)=f^{\prime \prime}\left(f^{-1}(z)\right) / f^{\prime}\left(f^{-1}(z)\right)^{2}$. Obviously $g$ is well defined and by Lemma 2 we have

$$
\lim _{k \rightarrow 1^{-}} \frac{f^{\prime \prime}(k)}{f^{\prime}(k)^{2}}=\lim _{h \rightarrow 0^{+}} g(h)=0 .
$$

Since $f$ is concave on $\left[f^{-1}\left(\frac{1}{2}\right), 1\right]$ we have

$$
g(u) \leq 0 \text { whenever } u \text { is in }\left(0, \frac{1}{2}\right]
$$

and by (7) and (8) for all $u, v$ in $(0,1)$ with $u+v<1$ we obtain

$$
g(u) \leq g(u+v)
$$

and

$$
g(u) \cdot g(v) \geq(g(u)+g(v)) \cdot g(u+v)
$$


Now we can apply Lemma 3 and conclude that $g$ must be the zero function, i.e., that $f^{\prime \prime}\left(f^{-1}(z)\right)=0$ for all $z$ in $(0,1)$. This in turn implies that $f$ must have the form $f(x)=a x+b$ for some constants $a, b$ and contradicts the assumption that $\lim _{x \rightarrow 0^{+}} f^{\prime}(x)=-\infty$.

ACKNOWLEDGMENTS. The authors thank Professor M. J. Frank (Illinois Institute of Technology, Chicago) for his interesting remarks concerning this paper and the referee for some helpful comments.

\section{BIBLIOGRAPHY}

1. J. Aczél, Lectures on functional equations and their applications, Academic Press, New York and London, 1966.

2. C. Alsina, On countable products of probabilistic metric spaces and algebraic convexifications, Pacific J. Math. 76 (1978), 291-300.

3. C. Alsina and B. Schweizer, The countable product of probabilistic metric spaces, Houston J. Math. 9 (1983), 303-310.

4. G. H. Hardy, J. E. Littlewood and G. Polya, Inequalities, Cambridge Univ. Press, Cambridge, 1934.

5. C. H. Ling, Representation of associative functions, Publ. Math. Debrecen 12 (1965), 189-212.

6. B. Schweizer and A. Sklar, Probabilistic metric spaces, Elsevier, New York, 1983.

Departament Matemàtiques I Estadística, Escola TÉcnica Superior D'Arquitectura, Universitat Politécnica de Catalunya, Avda Diagonal, 649, 08028 BARCELONA, SPAIN 